\title{
MRS Bulletin names Raman as Postdoctoral Publication Prize recipient
}

mrs.org/bulletin-prize

$\mathrm{T}$ he Materials Research Society (MRS) Bulletin has named Ritu Raman as the 2020 MRS Bulletin Postdoctoral Publication Prize recipient. This award recognizes postdoctoral researchers for their intellectual merit, the impact of their research and scholarship, and their interest in science writing and communications.

Raman was selected from a group of 94 highly qualified applicants and nominations "for her diverse research experience, outstanding academic achievements, high quality of scientific publications, extensive outreach efforts, and dedication to mentorship and professional service."

Raman currently is a postdoctoral fellow at the Massachusetts Institute of Technology (MIT) in the research group of Robert Langer. She is a National Academies of Sciences, Engineering, and Medicine Ford Foundation Postdoctoral Fellow (2019-2020) and a Koch Institute Postdoctoral Fellow (2019-2020). She was also the AAAS L'Oréal USA for Women in Science Fellow (2018-2019). Raman earned her $\mathrm{PhD}$ degree in mechanical engineering in 2016 from the University of Illinois at Urbana-Champaign and her BS degree in mechanical engineering in 2012 from Cornell University.

"As a biohybrid engineer, my scientific goal is to understand how we can build with living and nonliving materials in synchrony and to build adaptive medical implants that dynamically sense and respond to the human body," said Raman. "I envision a world where biological building blocks, like living cells, are part of the toolbox of every materials scientist, but this requires engaging and accessible science communication."

Biological materials can dynamically sense and adapt to their surroundings. Biohybrid design, the science of integrating biological and bioinspired materials with engineered systems, could thus yield machines capable of complex behaviors, such as self-assembly and self-healing.
These behaviors are especially desirable in devices that interface with the human body. "There is a compelling case for using biohybrid and biomimetic machines in the human body, because it is a highly variable and dynamic environment," said Raman.

As a postdoctoral fellow at MIT, Raman designed synthetic materials that mimic the adaptive behaviors of biological materials, implemented them in implantable devices, and validated their function in a range of in vivo models. She developed a light-triggerable tough hydrogel that dynamically actuates and degrades implantable devices without the need for invasive interventions, and demonstrated functionality in the gastrointestinal tract of a porcine model. She also developed a bidirectional nanofluidic pump, actuated by a heat-triggerable material, which performs label-free liquid biopsies of neural interstitial fluid and demonstrated functionality in the brain of a rodent model.

In addition to publishing many research and review papers, Raman is currently completing her first book, titled Biofabrication (MIT Press, expected publication in fall 2021). It covers the fundamental technology that enables building with biology and shares applications of biofabrication, such as tissue engineering, organs on a chip, biofabricated meat and leather, and biohybrid robotics.

While authoring the book, Raman was intentionally mindful about highlighting researchers from groups traditionally underrepresented in science. "Science should value diversity. We have so many hard problems to fix in our w orld. If you discourage half the people who could work on these problems, you are doing the world a huge disservice."

Raman also serves as an AAAS IF/ THEN ambassador, one of 125 women from around the country chosen to represent the diverse and exciting careers of women in science. IF/THEN hosts the largest free collection of authentic and relatable images

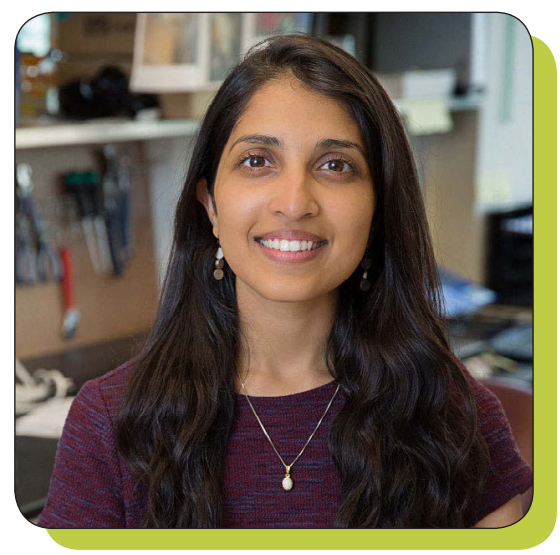

and videos of women in STEM. The goal is to inspire young girls to pursue STEM careers while creating a culture shift in how the world perceives women in the field.

When asked what advice she would give to young researchers, Raman emphasized diversifying research projects. "Think about research in the way a financial investor would think about their portfolio. Work on a few different things. Pressure test each to see their potential and strategically spend time on the highest value projects. Judge that value by the impact the project could have on the world - that will keep you persevering in the face of repeated failure, but also know when to cut your losses when you aren't getting anywhere."

Regarding writing, she recommends practice in person and on paper. "Talk to people who aren't scientists. Talk to them all day all the time. This will teach you to learn and respect different perspectives and write in a way that conveys meaning to a variety of audiences.

"Science is a team sport that relies on many types of expertise, and the emerging field of biohybrid design requires input from experts in the materials community. As I advance in my career, I plan on leveraging the MRS community as a central resource for learning and sharing the latest advances in biohybrid materials and machines."

The prize consists of a $\$ 2,000$ cash award, a profile published in MRS Bulletin, a complimentary one-year membership in the Materials Research Society, and an invitation to publish in MRS Bulletin.

MRS acknowledges the Jiang Family Foundation and MTI Corporation for their generous contributions to support the Postdoctoral Publication Prize. 